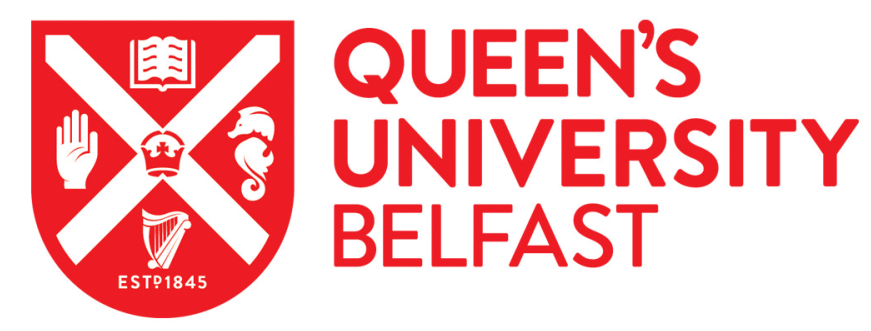

\title{
Rotational superstructure in van der Waals heterostructure of self- assembled C60 monolayer on the WSe2 surface
}

Santos, E., Scullion, D., Chu, X. S., Li, D. O., Guisinger, N. P., \& Wang, Q. H. (2017). Rotational superstructure in van der Waals heterostructure of self-assembled C60 monolayer on the WSe2 surface. Nanoscale, 9(35), 13245-13256. https://doi.org/10.1039/C7NR03951D

\section{Published in:}

Nanoscale

\section{Document Version:}

Peer reviewed version

Queen's University Belfast - Research Portal:

Link to publication record in Queen's University Belfast Research Portal

Publisher rights

Copyright 2017 Royal Society Of Chemistry. This work is made available online in accordance with the publisher's policies. Please refer to any applicable terms of use of the publisher.

\section{General rights}

Copyright for the publications made accessible via the Queen's University Belfast Research Portal is retained by the author(s) and / or other copyright owners and it is a condition of accessing these publications that users recognise and abide by the legal requirements associated with these rights.

Take down policy

The Research Portal is Queen's institutional repository that provides access to Queen's research output. Every effort has been made to ensure that content in the Research Portal does not infringe any person's rights, or applicable UK laws. If you discover content in the Research Portal that you believe breaches copyright or violates any law, please contact openaccess@qub.ac.uk. 


\section{Rotational Superstructure in van der Waals}

\section{Heterostructure of Self-Assembled $\mathrm{C}_{60}$ Monolayer on the $\mathrm{WSe}_{2}$ Surface}

Elton J. G. Santos, ${ }^{* \dagger}$ Declan Scullion,$^{\dagger}$ Ximo S. Chu,$^{\ddagger}$ Duo O. Li, ${ }^{\ddagger}$ Nathan P.

School of Mathematics and Physics, Queen's University Belfast, BT7 1NN, UK, Materials Science and Engineering, School for Engineering of Matter, Transport and Energy, Arizona State University, Tempe, Arizona 85287, USA, and Center for Nanoscale Materials, Argonne National Laboratory, Argonne, IL 60439, USA

E-mail: e.santos@qub.ac.uk; qhwang@asu.edu

* To whom correspondence should be addressed

${ }^{\dagger}$ School of Mathematics and Physics, Queen's University Belfast, BT7 1NN, UK

${ }^{\ddagger}$ Materials Science and Engineering, School for Engineering of Matter, Transport and Energy, Arizona State University, Tempe, Arizona 85287, USA

`Center for Nanoscale Materials, Argonne National Laboratory, Argonne, IL 60439, USA 


\begin{abstract}
Hybrid van der Waals (vdW) heterostructures composed of two-dimensional (2D) layered materials and self-assembled organic molecules are promising systems for electronic and optoelectronic applications with enhanced properties and performance. Control of molecular assembly is therefore paramount to fundamentally understand the nucleation, ordering, alignment, and electronic interaction of organic molecules with 2D materials. Here, we report the formation and detailed study of highly ordered, crystalline monolayers of $\mathrm{C}_{60}$ molecules self-assembled on the surface of $\mathrm{WSe}_{2}$ in wellordered arrays with large grain sizes $(\sim 5 \mu \mathrm{m})$. Using high-resolution scanning tunneling microscopy (STM), we observe a periodic $2 \times 2$ superstructure in the $\mathrm{C}_{60}$ monolayer and identify four distinct molecular appearances. Using vdW-corrected ab initio density functional theory (DFT) simulations, we determine that the interplay between vdW and Coulomb interactions as well as adsorbate-adsorbate and adsorbate-substrate interactions results in specific rotational arrangements of the molecules forming the superstructure. The orbital ordering through the relative positions of bonds in adjacent molecules create a charge redistribution that links the molecule units in a long-range network. This rotational superstructure extends throughout the self-assembled monolayer and opens a pathway towards engineering aligned hybrid organic/inorganic vdW heterostructures with 2D layered materials in a precise and controlled way.
\end{abstract}

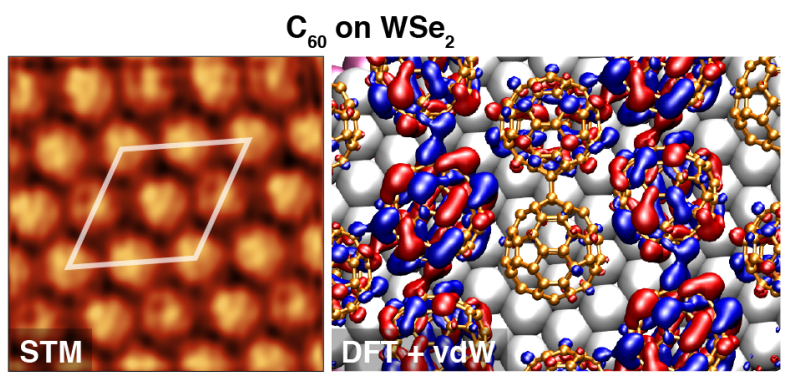

Graphical abstract density functional theory 


\section{Introduction}

The intense development of two-dimensional (2D) materials in recent years has expanded into the study of heterostructures formed using 2D layers and other materials. ${ }^{1-4}$ Heterostructures of different materials held together by van der Waals (vdW) forces allow materials of diverse compositions, structures, and properties to be combined, resulting in engineered materials with properties that are combinations of the components' properties, as well as newly emergent behaviours at the interfaces. Such heterostructures have been demonstrated using stacks of 2D layered materials ${ }^{14}$ and 2D materials combined with nanostructures of other dimensionalities and with organic crystals. ${ }^{[5]}$ The atomic flatness and lack of dangling bonds at the surface of 2D layered materials like graphene, boron nitride, and the transition metal dichalcogenides (TMDCs) allow them to form non-covalent interactions with a wide range of materials without the requirements for lattice matching that covalently bonded systems would have. Heterostructures of 2D layers can be achieved by physically stacking different sheets together or by epitaxial growth of subsequent $2 \mathrm{D}$ materials. ${ }^{1 / 46 / 7}$ At the interfaces between disparate materials, effects like charge transfer, tunneling, disorder, and impurity states can influence the electronic and optical behaviours. ${ }^{8}$ The careful combination of materials has resulted in new developments in performance and properties in devices like transistors, solar cells, and light emitting diodes. .51910

The vdW heterostructures formed by organic crystals on 2D layered materials generally take advantage of the atomically flat and chemically inert surfaces to template self-assembly of the molecule units into ordered arrangements.5111-17 Organic molecules that are often used in organic electronics, which typically have conjugated $\pi$-electron systems for better intermolecular conduction,, 18 have improved stacking and ordering when they are assembled by $2 \mathrm{D}$ materials. ${ }^{16}$ This has resulted in devices with significant increases in carrier mobility in field-effect transistors ${ }^{511920}$ and increased charge separation in photovoltaics. ${ }^{21}$ There are also promising opportunities for organic/2D vdW heterostructures to be used in flexible 
electronics. 224 The synergy between the mechanical robustness of the $2 \mathrm{D}$ layers and their diverse available electronic properties, ranging from semi-metals (e.g. graphene, silicene, germanene) to semiconductors (e.g. transition metal dichalcogenides (TMDCs)), $\stackrel{25}{ }$ combined with the chemical tunability of the molecules can open the door for further design rules based on organic/vdW heterostructures. There is a need to develop a fundamental understanding of how molecular processes happening at the early stage of the crystallization of organic molecules on $2 \mathrm{D}$ crystals drive the system to specific epitaxial relationships 11 and unique interfacial properties such as polymorphism. 12 Moreover, the control of two-dimensional self-assembly of single-layer of molecules on layered materials in terms of molecular ordering, alignment, and crystallinity can result in emergent behavior and exciting new physics. Therefore, it is essential to achieve a deep understanding of the basic physical and chemical phenomena that rule highly crystalline architectures involving crystals of organic molecules and 2D materials in potential device platforms.

Here we report the growth of high quality self-assembled monolayers of $\mathrm{C}_{60}$ on $\mathrm{WSe}_{2}$ as an example of a weakly interacting organic/2D vdW heterostructure system. $\mathrm{C}_{60}$ has been extensively used in the organic electronics field, and $\mathrm{WSe}_{2}$ is an important semiconducting 2D material. We study the interfacial properties of this system using complementary methods of high resolution scanning tunneling microscopy (STM) and $a b$ initio density functional theory including vdW interactions. $\mathrm{C}_{60}$ plays an important role as an acceptor in organic photovoltaics $(\mathrm{OPVs})^{2627}$ due to its high electron affinity for charge harvesting processes, and is expected to be similarly useful in hybrid $2 \mathrm{D} /$ organic optoelectronics. ${ }^{28}$ The interfacial interaction of $\mathrm{C}_{60}$ with other $2 \mathrm{D}$ TMDCs has been shown to result in doping, with pdoping occurring for $\mathrm{WSe}_{2}$ in particular, 29 and with graphene has led to charge transfer and increased carrier mobility. ${ }^{30}$ While the self-assembly of $\mathrm{C}_{60}$ molecules on metal surfaces

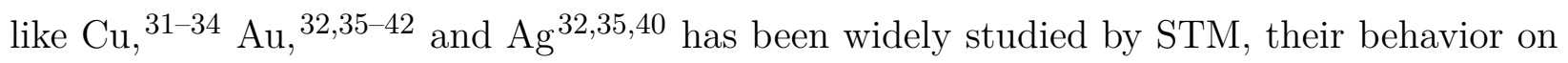
2D material substrates is relatively unknown aside from some studies on graphene. $15,43,46$ The electronic and physical structure of the substrate has played an important role in these 
earlier works, and is also expected to be crucial in the case of $\mathrm{WSe}_{2}$. Generally, there is a higher degree of charge transfer between metals and molecules than between 2D materials and molecules.

Our STM images reveal that $\mathrm{C}_{60}$ self-assembles into a close-packed monolayer on the surface of $\mathrm{WSe}_{2}$ that extends uniformly in islands as large as $\sim 5 \mu \mathrm{m}$. The long-range ordering and large grains we observe contrast with much smaller grains and local ordering seen in previous studies. This $\mathrm{C}_{60}$ monolayer exhibits four distinct intramolecular patterns in a $2 \times 2$ superlattice, which is unusual for a monolayer assembly. High-throughput first-principles calculations show that only a few molecular configurations are energetically favorable for $\mathrm{C}_{60}$ arranged on $\mathrm{WSe}_{2}$. The relative orientation of pentagons and hexagons between neighboring molecules drives the different arrangements through charge reordering connecting the $\mathrm{C}_{60}$ molecules in a periodic network. Moreover, a systematic increase of the charge transfer between $\mathrm{WSe}_{2}$ and $\mathrm{C}_{60}$ is observed as a function of short rotations of $\mathrm{C}_{60}$ mediated by vdW interactions. The increase in electron transfer goes along with the increase in stability of molecular configuration. This observation points to the active role of the molecule-substrate interactions in the stabilization of the interface. This also indicates that the presence of $\mathrm{C}_{60}$ has only a mild effect on the physical and electronic properties of $\mathrm{WSe}_{2}$ (e.g. electronic band gap, W-Se bond length, flatness), even though the molecules are electronically correlated. The creation of a clean interface between $\mathrm{WSe}_{2}$ and $\mathrm{C}_{60}$ resulting in a unique rotational superlattice is an intriguing step in the understanding and engineering of organic/2D vdW heterojunction devices.

\section{Results and discussion}

\section{Formation of self-assembled $\mathrm{C}_{60}$ monolayer}

The assembly of $\mathrm{C}_{60}$ on $\mathrm{WSe}_{2}$ was experimentally implemented by in situ thermal deposition of $\mathrm{C}_{60}$ in an ultrahigh vacuum (UHV) system and characterized by scanning tunneling 
microscopy (STM). A single-crystal $\mathrm{WSe}_{2}$ substrate was cleaved by scotch tape to expose a clean surface immediately before being introduced into the vacuum chamber for characterization and thermal deposition of $\mathrm{C}_{60}$. $\mathrm{STM}$ images of the clean $\mathrm{WSe}_{2}$ surface are shown in Fig. 1 1 a-b. The atomic structure of the $\mathrm{WSe}_{2}$ lattice is clearly visible in both images, with a triangular symmetry due to the alternating positions of the Se atoms at the surface. Two point defects are seen in Fig. 1 $\mathbf{a}$, and some undulation of the surface in Fig. 1b. Fullerene molecules $\left(\mathrm{C}_{60}\right)$ were thermally evaporated in situ onto the $\mathrm{WSe}_{2}$ surface held at room temperature. The deposition time was calibrated such that we achieved sub-monolayer coverage of $\mathrm{C}_{60}$ molecules. A schematic illustration of the $\mathrm{C}_{60}$ molecules on top of $\mathrm{WSe}_{2}$ is shown in Fig. 1c.

The sample was then cooled to $55 \mathrm{~K}$ for STM imaging, which showed that the fullerenes self-assemble into a close-packed hexagonal layer on $\mathrm{WSe}_{2}$, as seen in Fig. 1d. Because the sample is well below room temperature, the thermal motion of the molecules is minimized so that they can form a stable island with clear boundaries and long-range ordering. We note that bulk $\mathrm{C}_{60}$ crystals, which pack in a face-centered cubic (fcc) lattice, have fewer rotational freedoms below $260 \mathrm{~K}$, and have their orientational alignments frozen below $90 \mathrm{~K} . \underline{47 / 48}$ In our experiments, the substrate is at room temperature during thermal deposition of $\mathrm{C}_{60}$, allowing sufficient energy for the molecules to rotate and interact. The entire $\mathrm{C}_{60} / \mathrm{WSe}_{2}$ system is then gradually cooled to $55 \mathrm{~K}$, so that the optimal molecular configurations are stabilized before STM imaging.

The apparent height of the molecules is about $1 \mathrm{~nm}$, as shown in the line profile labeled ' 1 '. This height is similar to that of a $\mathrm{C}_{60}$ monolayer grown on $\mathrm{NaCl}$ crystals on $\mathrm{Au}(111)$, and is higher than the $\sim 0.6-0.7 \mathrm{~nm}$ observed for $\mathrm{C}_{60}$ on $\mathrm{Au}(111) . \stackrel{38}{ }$ The inset of Fig. $1 \mathbf{d}$ shows a $2 \mathrm{D}$ fast Fourier transform $(\mathrm{FFT})$ of the $\mathrm{C}_{60}$ region, with sharp points in a hexagonal pattern. The distance from the center to each point is approximately $1.0 \mathrm{~nm}^{-1}$, corresponding to a periodicity of approximately $1.0 \mathrm{~nm}$ between molecules. This close-packed arrangement of the $\mathrm{C}_{60}$ on $\mathrm{WSe}_{2}$ is similar to its arrangement on other substrates such as graphene, 15 [49 

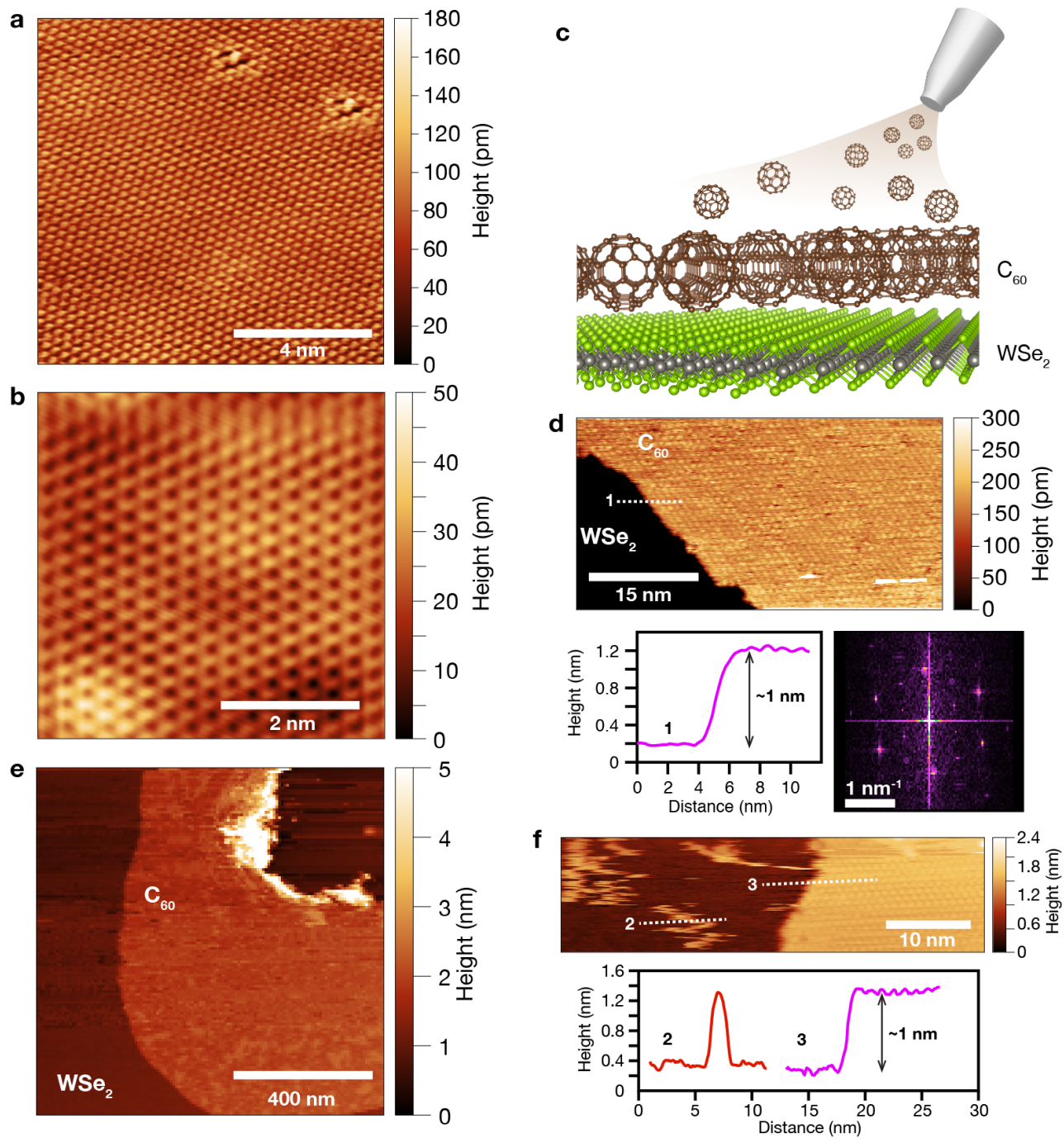

Figure 1: Self-assembly of $\mathbf{C}_{60}$ on $\mathbf{W S e}_{2}$. (a)-(b) Scanning tunneling microscopy (STM) images of mechanically exfoliated $\mathrm{WSe}_{2}$. The atomic lattice is visible in both images, along with two point defects in (a) and some local height variations in (b). Imaging conditions for (a) and (b): $0.6 \mathrm{~V}$ sample bias, $1 \mathrm{nA}$ tunneling current setpoint, $55 \mathrm{~K}$ sample temperature. (c) Schematic illustration of monolayer of $\mathrm{C}_{60}$ deposited by thermal evaporation and self-assembled on $\mathrm{WSe}_{2}$ surface. (d) STM image of $\mathrm{C}_{60}$ self-assembled monolayer island on $\mathrm{WSe}_{2}$. The flat $\mathrm{WSe}_{2}$ surface at the lower left of image appears very dark because the height scale has been adjusted to show periodicity in the $\mathrm{C}_{60}$ molecules. Below: Line profile along dashed line 1 , showing height of $\mathrm{C}_{60}$ molecules is $1 \mathrm{~nm}$, and 2D FFT of the $\mathrm{C}_{60}$ molecular arrangement, showing the points corresponding to a hexagonal pattern. Imaging conditions for (d): $2.5 \mathrm{~V}$ sample bias, $0.1 \mathrm{nA}$ tunneling current setpoint, $55 \mathrm{~K}$ sample temperature. (e) STM image showing larger area of $\mathrm{C}_{60}$ island on $\mathrm{WSe}_{2}$. Imaging conditions: $3.0 \mathrm{~V}$ sample bias, $0.1 \mathrm{nA}$ tunneling current. (f) STM image of the edge of a submonolayer island of $\mathrm{C}_{60}$, showing some molecules moving away from the edge at the left. The heights of the loose molecules and the rest of the island are the same, suggesting that the observed orderly arrangements are monolayers rather than bilayers. Imaging conditions: $2.2 \mathrm{~V}$ sample bias, $0.05 \mathrm{nA}$ tunneling current. 
$\mathrm{Au}, \stackrel{4142}{, 4}$ and $\mathrm{Cu} \cdot \frac{46}{\sqrt{6}}$ It is also similar to the (111) cut through the bulk fcc $\mathrm{C}_{60}$ crystal. In contrast, on reactive surfaces with dangling bonds such as $\mathrm{Si}$ and $\mathrm{SiC}, \mathrm{C}_{60}$ forms covalent bonds with the surface and do not form well-ordered layers,,$\frac{50}{52}$ although multilayers of $\mathrm{C}_{60}$ can form ordered lattices. $\frac{53}{53}$

We observe large islands of $\mathrm{C}_{60}$ (Fig. 1e) with dimensions up to $\sim 5 \mu \mathrm{m}$. In contrast, molecular islands of $\mathrm{C}_{60}$ on other substrates in the literature tend to be less than $100 \mathrm{~nm}$ in diameter. $15138 \mid 46$ We observe some instances of molecules freely moving across the WSe 2 surface with the same apparent $1 \mathrm{~nm}$ height, such as the ones in the line profile labeled ' 2 ' in Fig. 1f, confirming that our molecular islands are indeed monolayers of $\mathrm{C}_{60}$ rather than bilayers. Line profile ' 3 ' is taken at the edge of a molecular island.

In the high-resolution STM images of Fig. 2, the individual $\mathrm{C}_{60}$ molecules appear to have submolecular structure, relating to the complex shape of the electronic orbitals in the molecule. The distance between adjacent $\mathrm{C}_{60}$ molecules is approximately $1.0 \mathrm{~nm}$ (see line profile in Supporting Information, Figure S3). We can identify four individual configurations of $\mathrm{C}_{60}$, as highlighted by the circles labeled i, ii, iii, and iv in Fig. 2a. Each of these molecules is enlarged and cropped in Fig. 2 $\mathbf{b}$ to more clearly show their distinct appearances. Since sample bias is $+2.0 \mathrm{~V}$ in these images, these orbitals are likely to correspond to empty states (lowest unoccupied molecular orbitals, LUMO).

To understand the variations of the appearance of each $\mathrm{C}_{60}$ molecule on $\mathrm{WSe}_{2}$, we have performed first-principles density functional theory (DFT) calculations taking into consideration van der Waals (vdW) dispersion forces (see Methods section and Supplementary Information for details). As described below in detail, we can identify the most likely interface geometry as indicated in each simulated STM image in Fig. 2b. We have simulated the STM images for more than ten different configurations of $\mathrm{C}_{60}$ on top of $\mathrm{WSe}_{2}$ (see Fig. S1 in Supplementary Materials), with their energies shown in Fig. 3 as discussed in more detail below. These configurations can be organized in three different sets of symmetries as described in terms of observed rotational symmetry of the orbital lobes, e.g. 2-, 3- and 5-fold. Each 


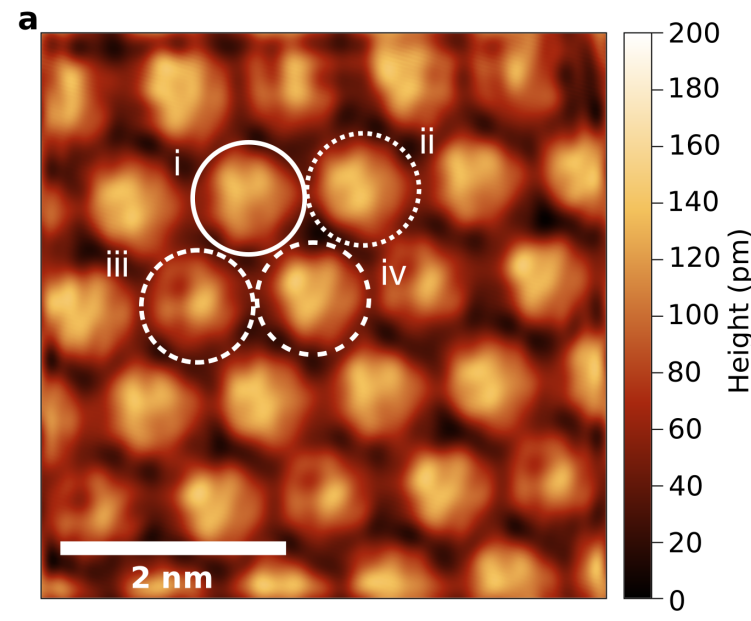

c i
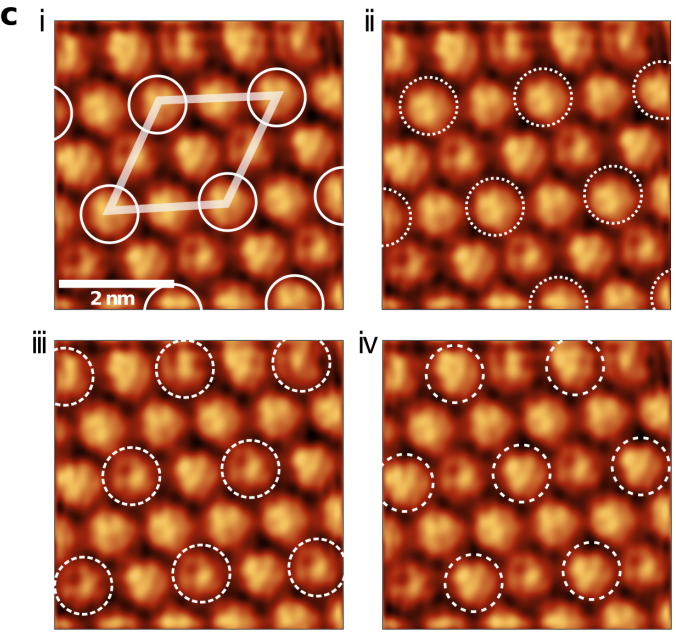

b
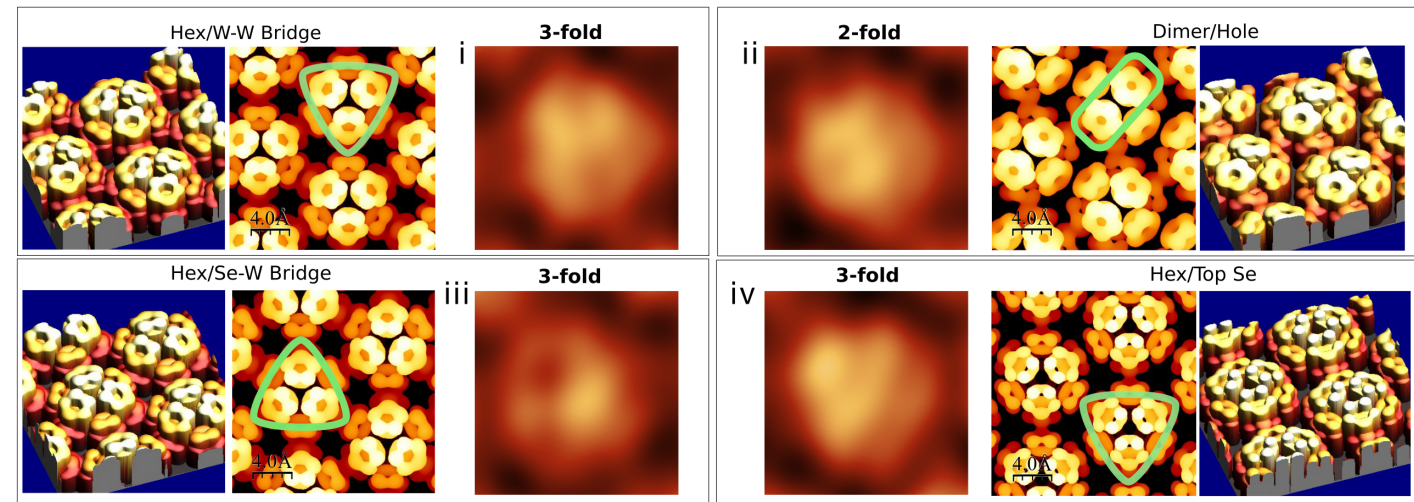

Figure 2: Molecular orientation superlattice of $\mathbf{C}_{60}$ on $\mathbf{W S e}_{2}$. (a) STM image of selfassembled monolayer of $\mathrm{C}_{60}$ molecules with submolecular resolution showing shapes of orbitals. Since the sample bias is $+2.0 \mathrm{~V}$, these are likely empty states (LUMO). The molecules are in a close-packed hexagonal arrangement. Four different orbital appearances are highlighted in the different circles, labeled i, ii, iii, iv, and are potentially attributed to different molecular orientations on the substrate surface. (b) Enlarged and cropped images of the four circled molecules from panel (a), showing their distinct appearances. Simulated STM images in 2D and 3D views are shown beside each panel with the corresponding interface geometries. The dominant symmetry $(2-, 3-$ fold) at each image is highlighted with green-outlines on the simulations. (c) The STM image from panel (a) is repeated here, with each of the four orbital appearances highlighted. They form a $2 \times 2$ superlattice arrangement as marked in panel i. Imaging conditions: $2.0 \mathrm{~V}$ sample bias, $0.2 \mathrm{nA}$ tunneling current setpoint, $55 \mathrm{~K}$ sample temperature. 
interfacial molecule seems to follow these symmetry rules even at the limit of full surface coverage. Indeed, looking closely at this limit we notice that these orbital appearances also form a $2 \times 2$ superlattice, as highlighted in Fig. $2 \mathrm{c}$. Each of the appearances i-iv is highlighted in each panel, with the circles indicating the repeated molecules. It is clear that each molecular appearance arises in the self-assembled monolayer of $\mathrm{C}_{60}$ every two molecules (Fig. 2 $\mathrm{c}$, panel i) to form a hexagonal pattern. In this $2 \times 2$ superlattice the distance between nearest neighboring molecules is $9.89 \AA$, which is close to the vdW distance in $\mathrm{C}_{60}$ bulk crystals. ${ }^{[54}$ We emphasize here that this $2 \times 2$ superlattice is observed in a monolayer of $\mathrm{C}_{60}$, while previous reports of orientational superlattices in $\mathrm{C}_{60}$ have been in bilayers on $\mathrm{Au}(111), \stackrel{42}{ }$ multilayers on $\mathrm{Cu}(111), \stackrel{55}{5}$ bulk $\mathrm{C}_{60}$ crystals,,$\frac{53}{}$ multilayers on $\mathrm{Ag}(111)$ with some local ordering, $\stackrel{56}{56}$ and bilayers and multilayers on $\mathrm{NaCl} / \mathrm{Au}(111) . \stackrel{38}{ }$ There have also been superstructures observed by STM for $\mathrm{C}_{60}$ on epitaxial graphene due to electronic Moiré patterns with either the $\mathrm{Ru}(0001)$ or $\mathrm{SiC}(0001)$ substrates rather than due to molecular rotations. .15143

\section{Configurations and rotations of $\mathrm{C}_{60}$ on $\mathrm{WSe}_{2}$}

To determine the effect of the interactions on the observed molecular patterns, we used $a b$ initio calculations at two different levels of theory with van der Waals interactions (DRSLL functional) and without (GGA, PBE functional). (See Methods section below and Supplementary Information for details.) We have initially considered a number of $\mathrm{C}_{60}$ molecular configurations on the $\mathrm{WSe}_{2}$ surface and calculated their electronic and energetic structures for a freestanding layer. A computational high-throughput screening taking into account the orientation of $\mathrm{C}$ atoms in the $\mathrm{C}_{60}$ molecule in pentagon, hexagon, dimer and apex positions, relative to the $\mathrm{WSe}_{2}$ structure, resulted in sixteen different arrangements as shown in Fig. 3. We clearly observed the role of $\mathrm{vdW}$ dispersion forces in the stabilization of the $\mathrm{C}_{60} / \mathrm{WSe}_{2}$ interfaces as we compare Fig. $3 \mathrm{a}$ and $3 \mathrm{~b}$. There is an enhancement of the stability in the vdW simulations as high as one order of magnitude relative to GGA results. The energy difference between the lowest and the highest stable configurations reaches $0.10 \mathrm{eV}$ and $0.30 \mathrm{eV}$ 
in GGA and vdW, respectively. We also observed that this increase in stability among the different configurations originates as $\mathrm{C}_{60}$ molecules partially rotate on the $\mathrm{WSe}_{2}$ surface, with the most stable configuration being the one where a hexagonal ring is on top of a Se atom (Hexagon/Top Se, top right of Fig. 3). This effect also influences the amount of charge transferred from $\mathrm{WSe}_{2}$ towards $\mathrm{C}_{60}$, which follows the vdW stability. As the interactions increase with more stability, the molecular orbitals of the $\mathrm{C}_{60}$ overlap more with the states at the surface, which increases the amount of charge transfer towards $\mathrm{C}_{60}$. That is, the more stable the configuration the more electron transfer. This is in accordance with the good acceptor characteristics of $\mathrm{C}_{60}$ due to its high electron affinity, which is advantageous

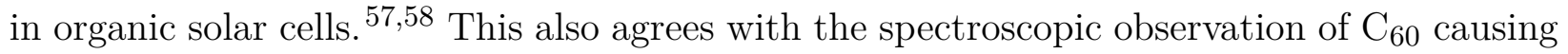
p-doping in $\mathrm{WSe}_{2} \cdot 29$

We now address the different relative orientations between the molecules in the $2 \times 2 \mathrm{su}-$ perlattice measured above (Fig. 2). It is well established that $\mathrm{C}_{60}$ molecules tend to perform rotations along some preferential directions when physisorbed on top of weakly interacting surfaces. $[59$ The strength of molecule-surface interactions and molecule-molecule forces determine the angular orientations of $\mathrm{C}_{60}$, which can vary as a function of temperature. When $\mathrm{C}_{60}$ is deposited on $\mathrm{WSe}_{2}$ surfaces, the molecules will have enough energy to perform molecular spinning, translations and some vibrations, e.g. breathing modes, as simulated using ab initio molecular dynamics at $55 \mathrm{~K}$ and $355 \mathrm{~K}$ (see Fig. 4, and movies in Supplementary Information). Most of the molecules perform short rotations in the first $1.0 \mathrm{ps}$, assuming different configurations relative to each other at later times. A constant spinning rate of all molecules has not been observed simultaneously for any initial configuration. Fig. 4 a shows the initial and final configurations after the system has time evolved for $10.5 \mathrm{ps.} \mathrm{The} \mathrm{relative}$ positions of the atoms of the $\mathrm{C}_{60}$ molecules are highlighted in blue and yellow to follow the evolution with time of the hexagonal and pentagonal rings, respectively, in each molecule. Interestingly, the molecular dynamics indicates that some molecules have their movement coupled to the nearest neighbors through interactions of double bonds localized between two 


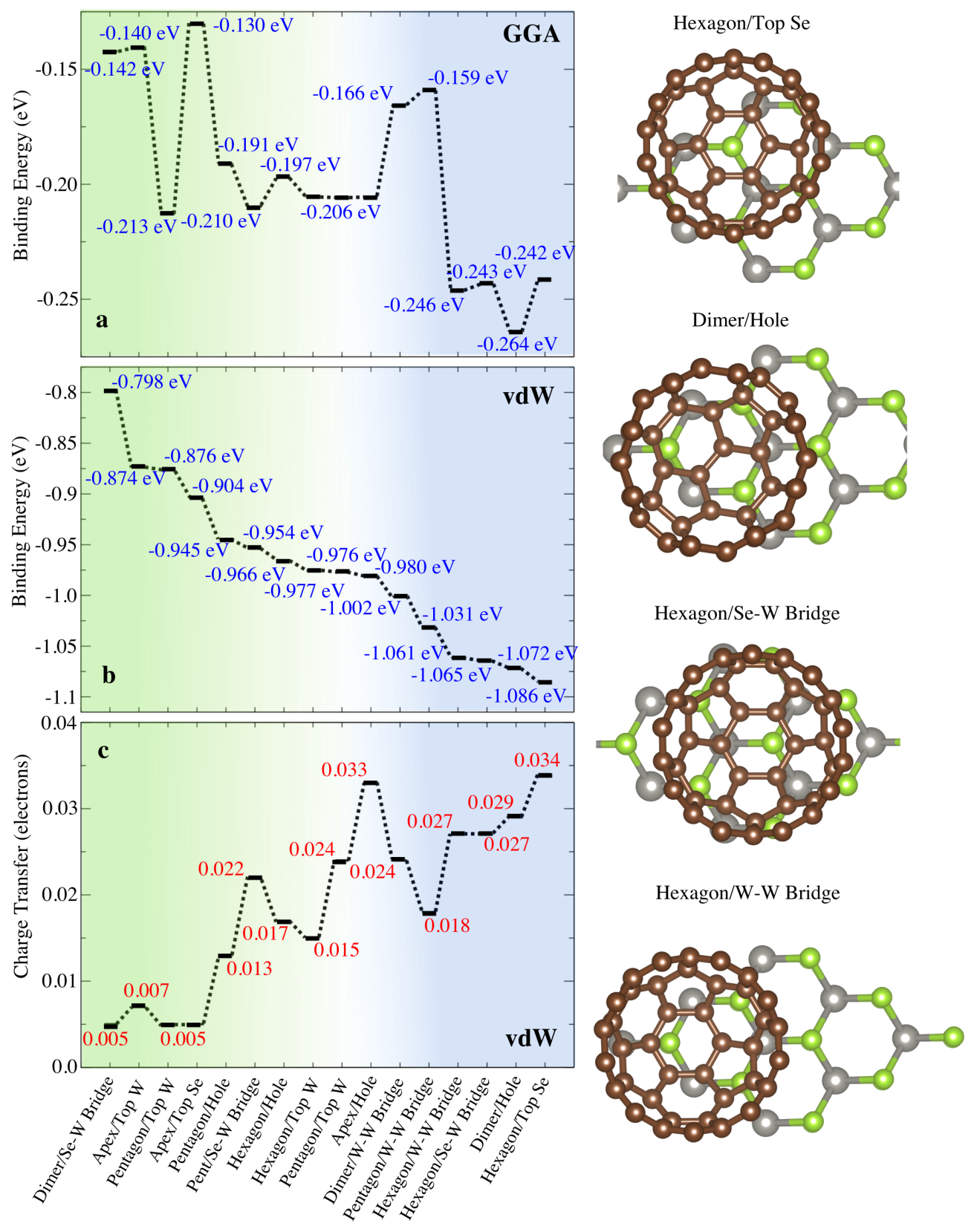

Figure 3: ab initio vdW electronic structure calculations. (a),(b) Calculated binding energies per $\mathrm{C}_{60}$ molecule at the level of GGA (PBE) and vdW (DRSLL) density functional theory, respectively, for a high-throughput computation screening of several configurations between $\mathrm{C}_{60}$ and $\mathrm{WSe}_{2}$. Configurations are ordered from the lowest to the highest bindings based on the vdW energy results. The four most stable molecular interfaces are shown on the right side in a top view perspective named accordingly to relative configuration of the $\mathrm{C}$ (brown), Mo (gray) and Se (green) atoms. For instance, a hexagonal ring in $\mathrm{C}_{60}$ might face the $\mathrm{WSe}_{2}$ surface in different ways, such as standing on top of a Se atom (Hexagon/Top Se ), or at a Se-W bridge position (Hexagon/Se-W bridge). (c) Interfacial charge transfer per molecule calculated at the level of vdW from $\mathrm{WSe}_{2}$ towards $\mathrm{C}_{60}$. 
hexagons (6:6) on one molecule and pentagonal faces of an adjacent $\mathrm{C}_{60}$ molecule (see movie 1 in Supplementary Materials). Such 6:6 bonds have a higher electronic density than bonds localized between a hexagon and pentagon (6:5) because of the local aromatic character. $\frac{5466}{56}$ This serves as an efficient point of interactions between the molecules.

\section{Electronic structure of hybrid $\mathrm{C}_{60} / \mathrm{WSe}_{2}$ system}

The energetic barriers for rotation between adjacent $\mathrm{C}_{60}$ molecules as a function of rotation angle $\theta$ (Fig. $4 \mathbf{b}$ ) show that the most stable positions occur at $0^{\circ}$ and $60^{\circ}$, which are angles where a 6:6 bond faces a pentagon (Fig. 4c). In this situation the high charge density of $p_{z}$ orbitals in 6:6 bonds overlap electron-poor pentagonal zones, which minimizes Coulomb interactions between molecules, therefore reducing the total energy of the system. The tendency for electron-rich and electron-poor regions of adjacent $\mathrm{C}_{60}$ molecules to associate has been also seen in previous reports. $\frac{38}{38}$ The wavefunctions of the conduction band at different rotational angles $\theta$ show the different orbital overlaps between the molecules (Fig. 4 d-e). In all configurations a substantial interaction is observed, with $\theta=30^{\circ}$ corresponding to two 6:6 bonds facing each other as the strongest (Fig. 4e). This configuration raises the energy by $\sim 334 \mathrm{meV}$ (Fig. $4 \mathbf{b}$ ), but provided an efficient coupling between $\mathrm{C}_{60}$ molecules as observed in the substantial charge density present throughout the entire system. Repulsive forces based on the short-range Pauli exclusion regime drive the system to short rotations where the strong overlap in wavefunctions between adjacent molecules can be tuned. It is noted that the charge density localized in the inter-molecule space clearly modifies its shape at different values of $\theta$, being more orbital-localized at low interactions energies (Fig. $4 \mathrm{~d}$ ), rather than spread between different molecules at high energy cost (Fig. 4e).

Furthermore, some meta-stable positions are also observed around $\theta=15^{\circ}$ and $\theta=45^{\circ}$, which are due to the stabilization of the charge arrangement between different $\mathrm{C}_{60}$ bonds; that is, $6: 5$ bonds and apex atoms in the $\mathrm{C}_{60}$. This suggests the directional nature of the $\mathrm{C}_{60}-\mathrm{C}_{60}$ interactions in the monolayer which acts as a driving force for organization and 
a
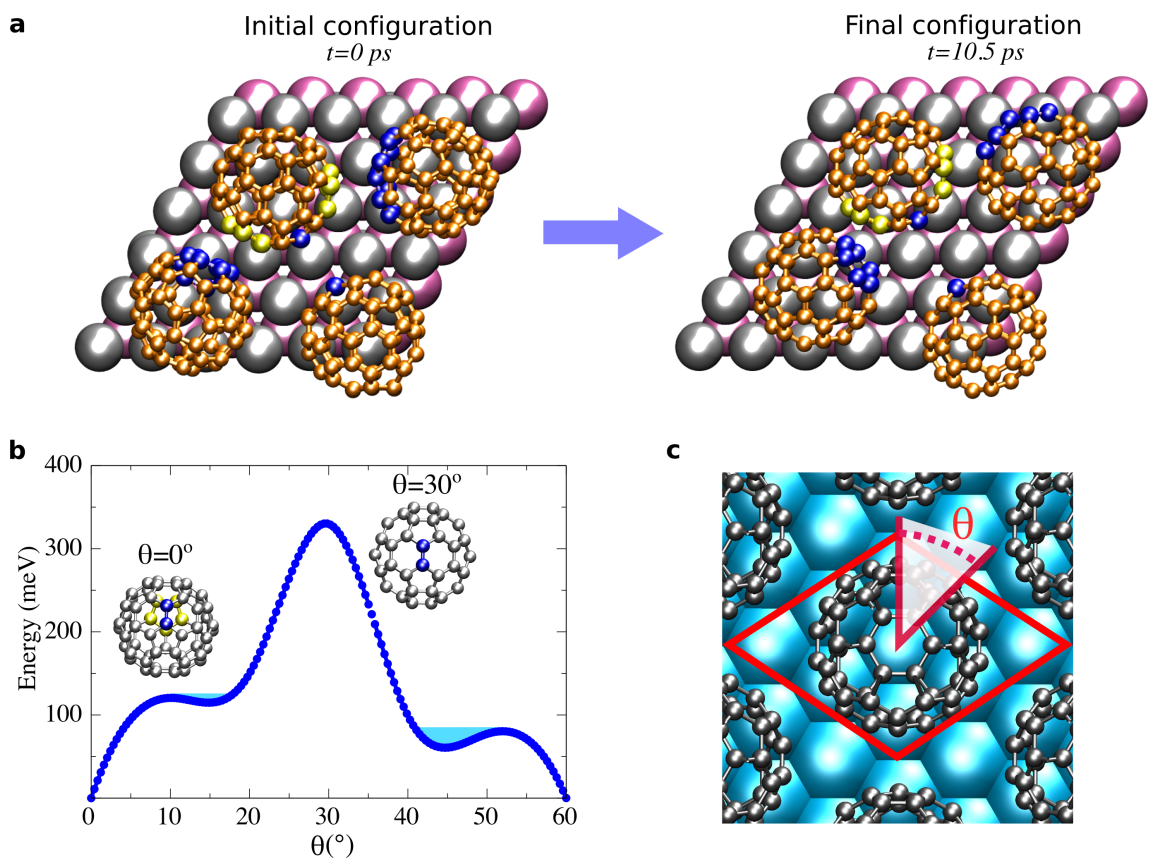

c

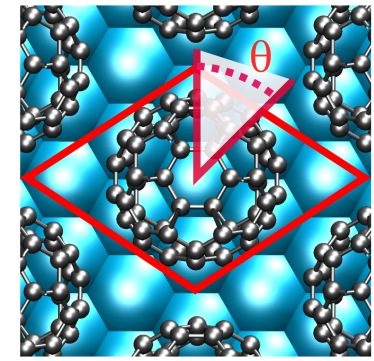

d

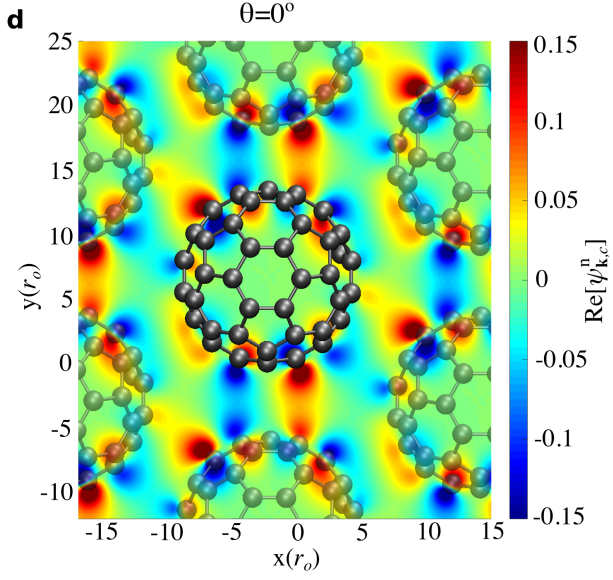

e

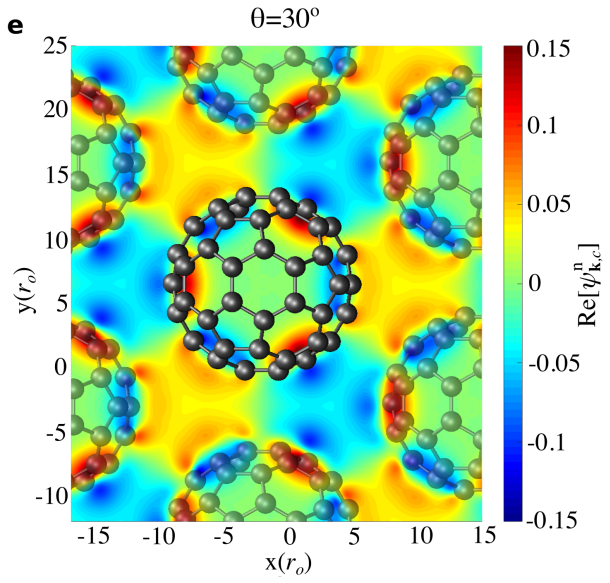

Figure 4: Molecular coupling in $\mathbf{C}_{60}$ molecules. (a) Ab initio molecular dynamics simulations including vdW dispersion forces for $\mathrm{C}_{60}$ molecules on $\mathrm{WSe}_{2}$. Atoms highlighted in blue (involving hexagons) and yellow (involving pentagons) tracked down the evolution of the molecules during the molecular dynamics where most of the interactions between the molecules happen. The system is set at $T=355 \mathrm{~K}$, and time-evolved for $t=10.5 \mathrm{ps}$. (b) Rotational barriers per interfacial molecule for $\mathrm{C}_{60}$ at the most stable configuration of Fig, $3 \mathbf{b}$ (Hexagon/Top Se). (c) Schematic of the unit cell and the definition of the rotational angle $\theta$ utilized in $\mathbf{b}$ relative to the next-neighbor molecules. The rotational angle $\theta$ is defined relative to the equator of the $\mathrm{C}_{60}$ molecule where spinning occurs along its center. Different angles correspond to distinct relative orientations between the $\mathrm{C}_{60}$ 's: $\theta=0^{\circ}$ (pentagon/6:6 configuration), $\theta=10^{\circ}$ (6:5/6:5 configuration), $\theta=30^{\circ}$ (6:6/6:6 configuration). Similar orientations are observed for $\theta>30$ because of the 3 -fold symmetry. (d-e) Cross section of the real part of the wave functions corresponding to the bottom of the conduction band $\psi_{\mathbf{k}, c}^{\mathrm{n}}$ at $\theta=0^{\circ}$ and $\theta=30^{\circ}$, respectively. Positive and negative values are shown in the color gradient map at the right of each panel. $\mathrm{C}$ atoms are shown in dark gray. 
self-assembly. Indeed, an estimation of the molecule-molecule interactions in the periodic two-dimensional $\mathrm{C}_{60}$ monolayer without the $\mathrm{WSe}_{2}$ gives a binding energy of $0.70 \mathrm{eV}(\theta=0)$, which is slightly smaller than those calculated between substrate and molecule at different adsorption configurations (Fig, $3 \mathrm{~b}$ ) but still in the same energy range. This indicates that some competition between molecule-molecule and molecule-surface interactions takes place at different values of $\theta$. In comparison to experiments performed on other surfaces, $\frac{42155}{4}$ where there are stronger interactions between $\mathrm{C}_{60}$ molecules and substrates, superlattices were only observed in bilayer islands likely due to the decoupling of the second layer from the substrate. In our experiments, the superlattice is observed in the $\mathrm{C}_{60}$ monolayer. If the balance between repulsion and attraction within the $\mathrm{C}_{60}$ monolayer is altered due to high molecule-surface interactions, similar patterns would not be observed. We emphasize that here in the case of $\mathrm{WSe}_{2}$ surfaces, the interactions are at just the right amount to permit the $\mathrm{C}_{60}$ molecules to spin and still be chemically coupled to the substrate.

The resulting electronic structure of the combined $\mathrm{C}_{60} / \mathrm{WSe}_{2}$ system after 10.5 ps of time evolution is shown in Fig. 5. The geometric configuration of the system is the one shown in Fig. 4 a. A band gap of about $0.50 \mathrm{eV}$ is clearly observed in the $\mathrm{C}_{60} / \mathrm{WSe}_{2}$ system, with contributions from conduction band $\mathrm{C}_{60}$ states (Fig. 5a). The bandgap of the $\mathrm{C}_{60}$ layer alone is close to $0.7 \mathrm{eV}$, which is considerably smaller than that of $\mathrm{C}_{60}$ packed in an FCC

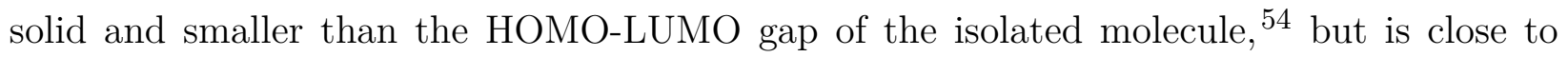
what was measured for $\mathrm{C}_{60}$ in a double barrier tunnel junction geometry ${ }^{67}$ This suggests that some delocalization of the $\mathrm{C}_{60}$ states throughout the entire system could be a key factor. Indeed, the real part of the fullerene wave functions, $\operatorname{Re}\left[\psi_{\mathbf{k}, c}^{\mathrm{n}}\right](\mathrm{n}=4,7)$, selected at the conduction band displayed such behavior (Fig. 5b-c). There is a remarkable electronic interaction between the $\mathrm{C}_{60}$ molecules which can be appreciated via the lateral extension of the molecular orbital linking the molecules in different spatial distributions. In fact, such orbital characteristics follow a molecular pattern that resembles the one observed in our STM measurements (Fig. 2). The spatial character of the wave function changes between 

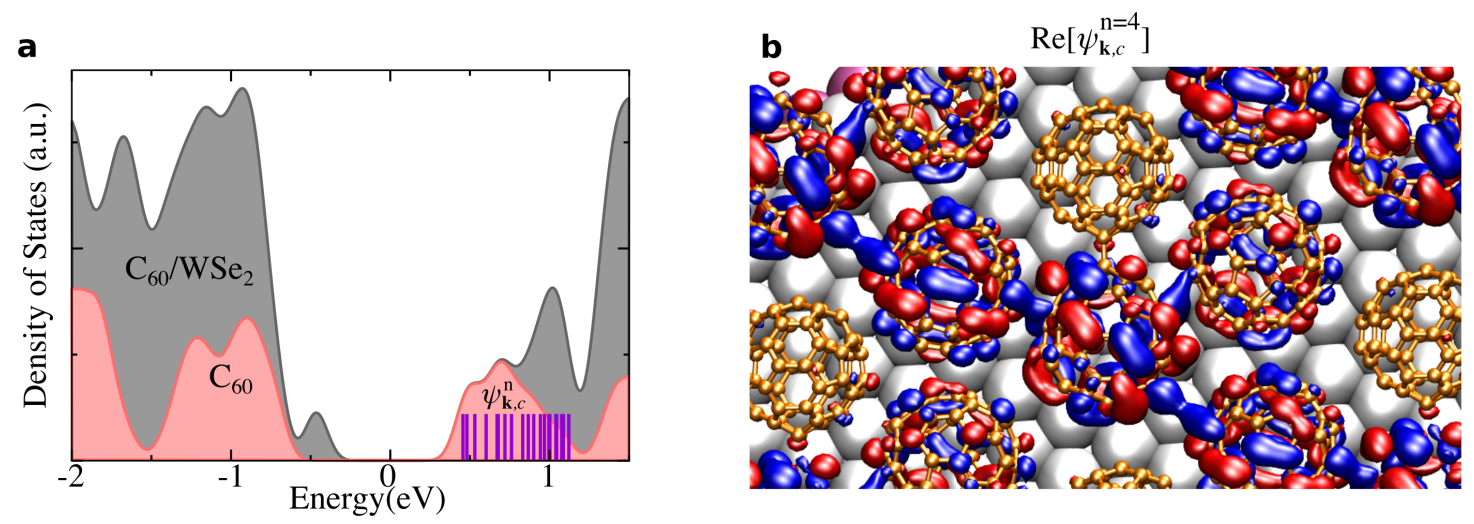

\section{d}
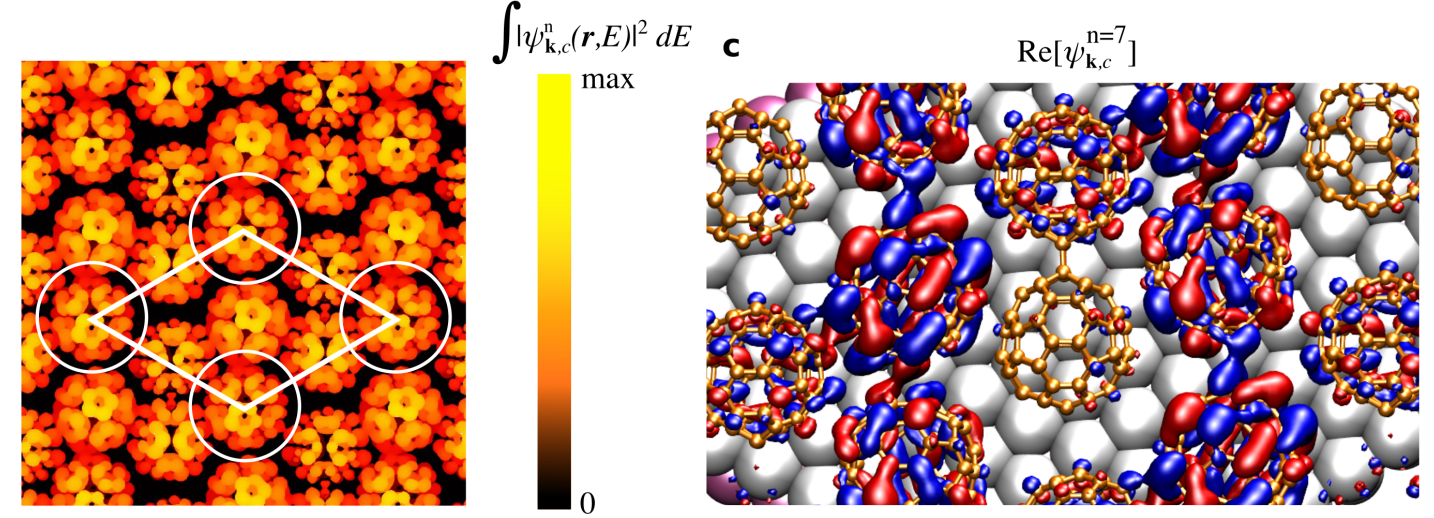

Figure 5: Electronic structure of $\mathbf{C}_{60} / \mathbf{W S e} \mathbf{e}_{2}$ heterostructure. (a) Density of states (DOS) of the $\mathrm{C}_{60} / \mathrm{WSe}_{2}$ heterostructure (gray) and $\mathrm{C}_{60}$ monolayer (pink) for the optimized geometry at $355 \mathrm{~K}$ using ab initio molecular dynamics simulations as shown in Fig. $4 \mathbf{a}$. The states at the bottom of the conduction band are shown individually through vertical bars with $\psi_{\mathbf{k}, c}^{\mathrm{n}}$, where $\mathrm{n}=1-19$. Fermi level is set to zero. (b-c) Isosurfaces $\left( \pm 0.001 \mathrm{e} / \mathrm{Bohr}^{3}\right)$ for the real part of the wave functions corresponding to the fourth and seventh eigenvalues, $\psi_{\mathbf{k}, c}^{\mathrm{n}=4,7}$ of the DOS represented in panel a. These eigenvalues are arbitrarily chosen to be representative ones from the full set of states at the conduction band. Most of the wave functions show similar interacting patterns between the molecules with slight variations between different $\psi_{\mathbf{k}, c}^{\mathrm{n}}$. Here we highlight only two of them, while other examples are shown in Fig. S2. Positive and negative parts of $\psi_{\mathbf{k}, c}^{\mathrm{n}}$ are shown in blue and red, respectively. Se, W and C atoms are shown in silver, pink and orange, respectively. (d) Local density of states plotted using the integration of all energy levels marked as $\psi_{\mathbf{k}, c}^{\mathrm{n}}(\mathrm{n}=1-19)$ in a. The periodicity of the $2 \times 2$ superlattice is highlighted for a particular $\mathrm{C}_{60}$ configuration in the supercell. 
one eigenvalue to another, not only for $\mathrm{n}=4,7$, but the orbital symmetry involved at each molecule is kept the same (see Fig. S2). In particular, all the eigenstates marked in Fig. 5 a at the bottom of the conduction band in the range of $0.45 \mathrm{eV}$ to $1.12 \mathrm{eV}$ inside of the band gap of the $\mathrm{WSe}_{2}$ surface have similar electronic characteristics. Integrating these states through:

$$
\rho(\mathbf{r}, E)=\int_{E_{o}}^{E_{o}+\epsilon}\left|\psi_{\mathbf{k}, c}^{\mathrm{n}}(\mathbf{r}, E)\right|^{2} d E
$$

gives their spatial distribution in terms of the local density of states as shown in Fig. 5 d. The quantity $\rho(\mathbf{r}, E)$ reproduces closely the main features observed in the measured STM images (Fig. 2), where every other molecule has the same molecular orbital distribution following a $2 \times 2$ superlattice. The precise combination of the $\mathrm{C}_{60}$ molecules in the supercell utilized can drive the system to different orientationally ordered $\mathrm{C}_{60}$ domains. However, once the main interactions between molecules and molecule-substrate take place the rotational superstructure is formed, even though the local molecular configuration of the individual $\mathrm{C}_{60}$ molecules might show differences. This is related with the dynamical aspect of the molecule itself associated with the collective character of the self-assembly.

\section{Conclusion}

In conclusion, our findings reveal fundamental knowledge of the physical and chemical phenomena of van der Waals heterostructures using self-assembled organic molecules and inorganic 2D materials, which have some subtle but important differences from self-assembly on metal surfaces. $\mathrm{C}_{60} / \mathrm{WSe}_{2}$ constitutes an archetypal vdW heterostructure with exciting possibilities for electronic devices based on atomically thin films. We have shown the self-assembly of $\mathrm{C}_{60}$ molecules on $\mathrm{WSe}_{2}$ layers via high-resolution STM and ab initio DFT including vdW interactions. After deposition, the molecules form a monolayer that extends uniformly over $\mathrm{WSe}_{2}$ with large grain sizes $(\sim 5 \mu \mathrm{m})$. The interplay and balance between 
adsorbate-adsorbate and adsorbate-substrate interactions leads to the formation of rotational arrays of self-assembled $2 \times 2$ molecules. Using the state-of-the-art vdW ab initio calculations, we demonstrate the critical role of the relative orientation between specific bonds in the $\mathrm{C}_{60}$ in the determination of the spatial superlattice. Through the minimization of the inter-molecule Coulomb interactions, the $\mathrm{C}_{60}$ molecules tend to be electronically coupled with long range orientational ordering, which is reflected in the high crystallinity of $\mathrm{C}_{60}$ on $\mathrm{WSe}_{2}$. The electronic structure of the hybrid system shows spatial delocalization of molecular orbitals throughout the $2 \times 2$ superlattice. The present study shows a mechanism of collective molecular restructuring based on the balance of non-covalent molecule--molecule and molecule-substrate interactions. These results may have implications in the geometrical control of the self-assembly of surface molecules for various electronic and optoelectronic applications based on vdW heterostructures of 2D materials. This highlights the advantages of organic vdW heterostructures over commonly used materials to achieve high-performance organic electronic devices, where such control of molecule assembly is not achievable. Future theoretical and experimental efforts will explore to what extent the surface-driven molecular self-assembly mechanism found here will influence the carrier mobility of the organic molecules, which is critical for device platforms.

\section{Methods}

\section{Fabrication of the $\mathrm{WSe}_{2} / \mathrm{C}_{60}$ heterostructure and measurements}

Ultrahigh vacuum scanning tunneling microscopy (UHV STM) imaging was conducted in an Omicron VT STM/AFM system. The $\mathrm{WSe}_{2}$ crystal (NanoScience Instruments) was cleaved by peeling away the top layer using adhesive tape in air, and then was immediately loaded into the vacuum system. The $\mathrm{C}_{60}$ molecules (Sigma Aldrich, sublimed, 99.9\%) were deposited in vacuum using a molecular beam thermal evaporator (Dodecon Nanotechnology GmbH) onto the $\mathrm{WSe}_{2}$ sample held at room temperature. STM probes were electrochemically etched 
$\mathrm{W}$ wire. The $\mathrm{WSe}_{2}$ sample with $\mathrm{C}_{60}$ monolayer was cooled to $55 \mathrm{~K}$ for all imaging. The STM electronics and software were from Nanonis, and the resulting images were processed with the Gwyddion software package. $\frac{68}{6}$ Image processing included low-pass noise removal and background flattening.

\section{vdW ab initio calculations}

Calculations were based on ab initio density functional theory using the SIESTA method 69 and the VASP code. ${ }^{70171}$ The generalized gradient approximation ${ }^{72}$ along with the DRSLL 73 functional was used in both methods, together with a double-zeta polarized basis set in SIESTA, and a well-converged plane-wave cutoff of $500 \mathrm{eV}$ in VASP. We used a Fermi-Dirac distribution with an electronic temperature of $\mathrm{k}_{B T}=20 \mathrm{meV}$. Additional details are provided in the Supporting Information.

\section{Acknowledgments}

Use of the Center for Nanoscale Materials, an Office of Science user facility, was supported by the U. S. Department of Energy, Office of Science, Office of Basic Energy Sciences, under Contract No. DE-AC02-06CH11357. E.J.G.S. acknowledges the use of computational resources from the UK national high performance computing service, ARCHER, for which access was obtained via the UKCP consortium and funded by EPSRC grant ref EP/K013564/1; and the Extreme Science and Engineering Discovery Environment (XSEDE), supported by NSF grants number TG-DMR120049 and TG-DMR150017. The Queen's Fellow Award through the startup grant number M8407MPH and the Energy Sustainable PRP (QUB) are also acknowledged. D.S. acknowledges the EPSRC studentship. Q.H.W. acknowledges support from Arizona State University startup funds. 


\section{Author Contributions}

Q.H.W. and E.J.G.S. designed the project, analyzed the data, and wrote the manuscript. E.J.G.S. and D.S. conducted the DFT calculations. Q.H.W., N.P.G., X.S.C., and D.O.L. conducted the STM experiments.

\section{Supporting Information}

Supporting Information Available: Additional details on calculation methods, simulated $\mathrm{STM}$ images of all $\mathrm{C}_{60}$ configurations on $\mathrm{WSe}_{2}$, wavefunctions of $\mathrm{C}_{60} / \mathrm{WSe}_{2}$, and molecular dynamics movies of $\mathrm{C}_{60}$ on $\mathrm{WSe}_{2}$.

\section{Notes}

The authors declare no competing financial interest.

\section{References}

1. Geim, A. K.; Grigorieva, I. V. Van der Waals heterostructures. Nature 2013, 499, 419425.

2. Lee, G.-H.; Yu, Y.-J.; Cui, X.; Petrone, N.; Lee, C.-H.; Choi, M. S.; Lee, D.-Y.; Lee, C.; Yoo, W. J.; Watanabe, K. et al. Flexible and Transparent $\mathrm{MoS}_{2}$ Field-Effect Transistors on Hexagonal Boron Nitride-Graphene Heterostructures. ACS Nano 2013, 7, 7931-7936.

3. Liu, Y.; Weiss, N. O.; Duan, X.; Cheng, H.-C.; Huang, Y.; Duan, X. Van der Waals heterostructures and devices. Nature Rev. Mater. 2016, 1, 16042.

4. Novoselov, K. S.; Mishchenko, A.; Carvalho, A.; Castro Neto, A. H. 2D materials and van der Waals heterostructures. Science 2016, 353, aac9439.

5. Lee, C.-H.; Schiros, T.; Santos, E. J. G.; Kim, B.; Yager, K. G.; Kang, S. J.; Lee, S.; Yu, J.; Watanabe, K.; Taniguchi, T. et al. Epitaxial Growth of Molecular Crystals on 
van der Waals Substrates for High-Performance Organic Electronics. Adv. Mater. 2014, 26, 2812-2817.

6. Withers, F.; Del Pozo-Zamudio, O.; Mishchenko, A.; Rooney, A. P.; Gholinia, A.; Watanabe, K.; Taniguchi, T.; Haigh, S. J.; Geim, A. K.; Tartakovskii, A. I. et al. Light-emitting diodes by band-structure engineering in van der Waals heterostructures. Nature Mater. 2015, 14, 301-306.

7. Jariwala, D.; Marks, T. J.; Hersam, M. C. Mixed-dimensional van der Waals heterostructures. Nature Mater. 2017, 16, 170-181.

8. Li, S.-L.; Tsukagoshi, K.; Orgiu, E.; Samori, P. Charge transport and mobility engineering in two-dimensional transition metal chalcogenide semiconductors. Chem. Soc. Rev. 2016, 45, 118-151.

9. Jeong, H.; Bang, S.; Oh, H. M.; Jeong, H. J.; An, S.-J.; Han, G. H.; Kim, H.; Kim, K. K.; Park, J. C.; Lee, Y. H. et al. Semiconductor-Insulator-Semiconductor Diode Consisting of Monolayer $\mathrm{MoS}_{2}$, h-BN, and GaN Heterostructure. ACS Nano 2015, 9, 10032-10038.

10. Lopez-Sanchez, O.; Alarcon Llado, E.; Koman, V.; Fontcuberta i Morral, A.; Radenovic, A.; Kis, A. Light Generation and Harvesting in a van der Waals Heterostructure. ACS Nano 2014, 8, 3042-3048.

11. Kim, K.; Lee, T. H.; Santos, E. J. G.; Jo, P. S.; Salleo, A.; Nishi, Y.; Bao, Z. Structural and Electrical Investigation of $\mathrm{C}_{60}$-Graphene Vertical Heterostructures. ACS Nano 2015, 9, 5922-5928.

12. Kim, K.; Santos, E. J. G.; Lee, T. H.; Nishi, Y.; Bao, Z. Epitaxially Grown Strained Pentacene Thin Film on Graphene Membrane. Small 2015, 11, 2037-2043.

13. Wang, Q. H.; Hersam, M. C. Room-temperature molecular-resolution characterization 
of self-assembled organic monolayers on epitaxial graphene. Nature Chem. 2009, 1, 206211.

14. Wang, Q. H.; Hersam, M. C. Nanofabrication of Heteromolecular Organic Nanostructures on Epitaxial Graphene via Room Temperature Feedback-Controlled Lithography. Nano Lett. 2011, 11, 589-593.

15. Cho, J.; Smerdon, J.; Gao, L.; Süzer, Ö.; Guest, J. R.; Guisinger, N. P. Structural and Electronic Decoupling of $\mathrm{C}_{60}$ from Epitaxial Graphene on SiC. Nano Lett. 2012, 12, 3018-3024.

16. Roy, S. S.; Bindl, D. J.; Arnold, M. S. Templating Highly Crystalline Organic Semiconductors Using Atomic Membranes of Graphene at the Anode/Organic Interface. J. Phys. Chem. Lett. 2012, 3, 873-878.

17. Zhang, L.; Roy, S. S.; Hamers, R. J.; Arnold, M. S.; Andrew, T. L. Molecular OrientationDependent Interfacial Energetics and Built-in Voltage Tuned by a Template Graphene Monolayer. J. Phys. Chem. C 2015, 119, 45-54.

18. Wang, C.; Dong, H.; Hu, W.; Liu, Y.; Zhu, D. Semiconducting $\pi$-Conjugated Systems in Field-Effect Transistors: A Material Odyssey of Organic Electronics. Chem. Rev. 2012, 112, 2208-2267.

19. Lee, T. H.; Kim, K.; Kim, G.; Park, H. J.; Scullion, D.; Shaw, L.; Kim, M.-G.; Gu, X.; Bae, W.-G.; Santos, E. J. G. et al. Chemical Vapor-Deposited Hexagonal Boron Nitride as a Scalable Template for High-Performance Organic Field-Effect Transistors. Chem. Mater. 2017, 29, 2341-2347.

20. Jariwala, D.; Howell, S. L.; Chen, K.-S.; Kang, J.; Sangwan, V. K.; Filippone, S. A.; Turrisi, R.; Marks, T. J.; Lauhon, L. J.; Hersam, M. C. Hybrid, Gate-Tunable, van der Waals p-n Heterojunctions from Pentacene and $\mathrm{MoS}_{2}$. Nano Lett. 2016, 16, 497-503. 
21. Bettis Homan, S.; Sangwan, V. K.; Balla, I.; Bergeron, H.; Weiss, E. A.; Hersam, M. C. Ultrafast Exciton Dissociation and Long-Lived Charge Separation in a Photovoltaic Pentacene-MoS 2 van der Waals Heterojunction. Nano Lett. 2017, 17, 164-169.

22. Briseno, A. L.; Mannsfeld, S. C. B.; Ling, M. M.; Liu, S.; Tseng, R. J.; Reese, C.; Roberts, M. E.; Yang, Y.; Wudl, F.; Bao, Z. Patterning organic single-crystal transistor arrays. Nature 2006, 444, 913-917.

23. Bao, Z.; Locklin, J. Organic Field-Effect Transistors; CRC Press, 2007.

24. Rogers, J. A.; Someya, T.; Huang, Y. Materials and Mechanics for Stretchable Electronics. Science 2010, 327, 1603-1607.

25. Fiori, G.; Bonaccorso, F.; Iannaccone, G.; Palacios, T.; Neumaier, D.; Seabaugh, A.; Banerjee, S. K.; Colombo, L. Electronics based on two-dimensional materials. Nat. Nanotechnol. 2014, 9, 768-779.

26. Krebs, F. C. Fabrication and processing of polymer solar cells: A review of printing and coating techniques. Sol. Energ. Mat. Sol. Cells 2009, 93, 394-412.

27. Yoo, S.; Domercq, B.; Kippelen, B. Efficient thin-film organic solar cells based on pentacene/ $\mathrm{C}_{60}$ heterojunctions. Appl. Phys. Lett. 2004, 85, 5427-5429.

28. Parui, S.; Pietrobon, L.; Ciudad, D.; Vélez, S.; Sun, X.; Casanova, F.; Stoliar, P.; Hueso, L. E. Gate-Controlled Energy Barrier at a Graphene/Molecular Semiconductor Junction. Adv. Func. Mater. 2015, 25, 2972-2979.

29. Osada, K.; Tanaka, M.; Ohno, S.; Suzuki, T. Photoinduced charge transfer from vacuumdeposited molecules to single-layer transition metal dichalcogenides. Jpn. J. Appl. Phys. 2016, 55, 065201. 
30. Jnawali, G.; Rao, Y.; Beck, J. H.; Petrone, N.; Kymissis, I.; Hone, J.; Heinz, T. F. Observation of Ground- and Excited-State Charge Transfer at the $\mathrm{C}_{60}$ /Graphene Interface. ACS Nano 2015, 9, 7175-7185.

31. Daughton, D. R.; Gupta, J. A. Orientation dependence of charge transfer for $\mathrm{C}_{60}$ on Cu(100). Appl. Phys. Lett. 2011, 98, 133303.

32. Gimzewski, J. K.; Modesti, S.; David, T.; Schlittler, R. R. Scanning tunneling microscopy of ordered $\mathrm{C}_{60}$ and $\mathrm{C}_{70}$ layers on $\mathrm{Au}(111), \mathrm{Cu}(111), \mathrm{Ag}(110)$, and $\mathrm{Au}(110)$ surfaces. $J$. Vac. Sci. Technol., B 1994, 12, 1942-1946.

33. Hashizume, T.; Motai, K.; Wang, X. D.; Shinohara, H.; Saito, Y.; Maruyama, Y.; Ohno, K.; Kawazoe, Y.; Nishina, Y.; Pickering, H. W. et al. Intramolecular structures of $\mathrm{C}_{60}$ molecules adsorbed on the $\mathrm{Cu}(111)-(1 \times 1)$ surface. Phys. Rev. Lett. 1993, 71, 2959-2962.

34. Larsson, J. A.; Elliott, S. D.; Greer, J. C.; Repp, J.; Meyer, G.; Allenspach, R. Orientation of individual $\mathrm{C}_{60}$ molecules adsorbed on $\mathrm{Cu}(111)$ : Low-temperature scanning tunneling microscopy and density functional calculations. Phys. Rev. B 2008, 77, 115434.

35. Altman, E. I.; Colton, R. J. Determination of the orientation of $\mathrm{C}_{60}$ adsorbed on $\mathrm{Au}(111)$ and $\operatorname{Ag}(111)$. Phys. Rev. B 1993, 48, 18244-18249.

36. Paßens, M.; Karthäuser, S. Interfacial and intermolecular interactions determining the rotational orientation of $\mathrm{C}_{60}$ adsorbed on $\mathrm{Au}(111)$. Surf. Sci. 2015, 642, 11-15.

37. Rogero, C.; Pascual, J. I.; Gómez-Herrero, J.; Baró, A. M. Resolution of site-specific bonding properties of $\mathrm{C}_{60}$ adsorbed on $\mathrm{Au}(111)$. J. Chem. Phys. 2002, 116, 832-836.

38. Rossel, F.; Pivetta, M.; Patthey, F.; Ćavar, E.; Seitsonen, A. P.; Schneider, W.-D. Growth and characterization of fullerene nanocrystals on $\mathrm{NaCl} / \mathrm{Au}(111)$. Phys. Rev. B 2011, 84, 075426. 
39. Tang, L.; Xie, Y.; Guo, Q. Complex orientational ordering of $\mathrm{C}_{60}$ molecules on $\mathrm{Au}(111)$. J. Chem. Phys. 2011, 135, 114702.

40. Wang, L.-L.; Cheng, H.-P. Density functional study of the adsorption of a $\mathrm{C}_{60}$ monolayer on $\operatorname{Ag}(111)$ and $\operatorname{Au}(111)$ surfaces. Phys. Rev. B 2004, 69, 165417.

41. Schull, G.; Berndt, R. Orientationally Ordered $(7 \times 7)$ Superstructure of $\mathrm{C}_{60}$ on $\mathrm{Au}(111)$. Phys. Rev. Lett. 2007, 99, 226105.

42. Tang, L.; Guo, Q. Orientational ordering of the second layer of $\mathrm{C}_{60}$ molecules on $\mathrm{Au}(111)$. Phys. Chem. Chem. Phys. 2012, 14, 3323-3328.

43. Zhou, H. T.; Mao, J. H.; Li, G.; Wang, Y. L.; Feng, X. L.; Du, S. X.; Müllen, K.; Gao, H.J. Direct imaging of intrinsic molecular orbitals using two-dimensional, epitaxiallygrown, nanostructured graphene for study of single molecule and interactions. Appl. Phys. Lett. 2011, 99, 153101.

44. Li, G.; Zhou, H. T.; Pan, L. D.; Zhang, Y.; Mao, J. H.; Zou, Q.; Guo, H. M.; Wang, Y. L.; Du, S. X.; Gao, H.-J. Self-assembly of $\mathrm{C}_{60}$ monolayer on epitaxially grown, nanostructured graphene on Ru(0001) surface. Appl. Phys. Lett. 2012, 100, 013304.

45. Švec, M.; Merino, P.; Dappe, Y. J.; González, C.; Abad, E.; Jelínek, P.; MartínGago, J. A. van der Waals interactions mediating the cohesion of fullerenes on graphene. Phys. Rev. B 2012, 86, 121407.

46. Jung, M.; Shin, D.; Sohn, S.-D.; Kwon, S.-Y.; Park, N.; Shin, H.-J. Atomically resolved orientational ordering of $\mathrm{C}_{60}$ molecules on epitaxial graphene on $\mathrm{Cu}(111)$. Nanoscale 2014, 6, 11835-11840.

47. Heiney, P. A. Structure, dynamics and ordering transition of solid $\mathrm{C}_{60}$. J. Phys. Chem. Solids 1992, 53, $1333-1352$. 
48. David, W. I. F.; Ibberson, R. M.; Dennis, T. J. S.; Hare, J. P.; Prassides, K. Structural Phase Transitions in the Fullerene $\mathrm{C}_{60}$. Europhys. Lett. 1992, 18, 219.

49. Li, G.; Zhou, H. T.; Pan, L. D.; Zhang, Y.; Mao, J. H.; Zou, Q.; Guo, H. M.; Wang, Y. L.; Du, S. X.; Gao, H.-J. Self-assembly of $\mathrm{C}_{60}$ monolayer on epitaxially grown, nanostructured graphene on $\mathrm{Ru}(0001)$ surface. Appl. Phys. Lett. 2012, 100, 013304.

50. Ovramenko, T.; Spillebout, F.; Bocquet, F. C.; Mayne, A. J.; Dujardin, G.; Sonnet, P.; Stauffer, L.; Ksari, Y.; Themlin, J. M. STM imagery and density functional calculations of $\mathrm{C}_{60}$ fullerene adsorption on the $6 \mathrm{H}-\mathrm{SiC}(0001)-3 \times 3$ surface. Phys. Rev. B 2013, 87, 155421.

51. Chen, D.; Sarid, D. An STM study of $\mathrm{C}_{60}$ adsorption on $\operatorname{Si}(100)-(2 \times 1)$ surfaces: from physisorption to chemisorption. Surf. Sci. 1995, 329, 206-218.

52. Pascual, J. I.; Gómez-Herrero, J.; Rogero, C.; Baró, A. M.; Sánchez-Portal, D.; Artacho, E.; Ordejón, P.; Soler, J. M. Seeing molecular orbitals. Chem. Phys. Lett. 2000, 321, 78-82.

53. Wang, H.; Zeng, C.; Wang, B.; Hou, J. G.; Li, Q.; Yang, J. Orientational configurations of the $\mathrm{C}_{60}$ molecules in the $2 \times 2$ superlattice on a solid $\mathrm{C}_{60}(111)$ surface at low temperature. Phys. Rev. B 2001, 63, 085417.

54. Dresselhaus, M.; Dresselhaus, G.; Eklund, P. Science of Fullerenes and Carbon Nanotubes; Academic Press, 1996.

55. Leaf, J.; Stannard, A.; Jarvis, S. P.; Moriarty, P.; Dunn, J. L. A Combined Monte Carlo and Hückel Theory Simulation of Orientational Ordering in $\mathrm{C}_{60}$ Assemblies. J. Phys. Chem. C 2016, 120, 8139-8147.

56. Große, C.; Gunnarsson, O.; Merino, P.; Kuhnke, K.; Kern, K. Nanoscale Imaging of 
Charge Carrier and Exciton Trapping at Structural Defects in Organic Semiconductors. Nano Lett. 2016, 16, 2084-2089, PMID: 26871739.

57. Clarke, T. M.; Durrant, J. R. Charge Photogeneration in Organic Solar Cells. Chem. Rev. 2010, 110, 6736-6767.

58. Santos, E. J. G.; Wang, W. L. Ultrafast charge-transfer in organic photovoltaic interfaces: geometrical and functionalization effects. Nanoscale 2016, 8, 15902-15910.

59. Hou, J. G.; Jinlong, Y.; Haiqian, W.; Qunxiang, L.; Changgan, Z.; Lanfeng, Y.; Bing, W.; Chen, D. M.; Qingshi, Z. Surface science: Topology of two-dimensional $\mathrm{C}_{60}$ domains. Nature 2001, 409, 304-305.

60. Sanchez-Sanchez, C.; Lanzilotto, V.; Gonzalez, C.; Verdini, A.; de Andres, P. L.; Floreano, L.; Lopez, M. F.; Martin-Gago, J. A. Weakly Interacting Molecular Layer of Spinning $\mathrm{C}_{60}$ Molecules on $\mathrm{TiO}_{2}(110)$ Surfaces. Chem. Eur. J. 2012, 18, 7382-7387.

61. Wang, Y.; Yamachika, R.; Wachowiak, A.; Grobis, M.; Crommie, M. F. Tuning fulleride electronic structure and molecular ordering via variable layer index. Nature Mater. 2008, 7, 194-197.

62. Chen, W.; Zhang, H.; Huang, H.; Chen, L.; Wee, A. T. S. Orientationally Ordered $\mathrm{C}_{60}$ on p-Sexiphenyl Nanostripes on Ag(111). ACS Nano 2008, 2, 693-698.

63. Altman, E. I.; Colton, R. J. Determination of the orientation of $\mathrm{C}_{60}$ adsorbed on $\mathrm{Au}(111)$ and $\mathrm{Ag}(111)$. Phys. Rev. B 1993, 48, 18244-18249.

64. Altman, E. I.; Colton, R. J. Nucleation, growth, and structure of fullerene films on $\mathrm{Au}(111)$. Surf. Sci. 1992, 279, 49-67.

65. Yuan, L.-F.; Yang, J.; Wang, H.; Zeng, C.; Li, Q.; Wang, B.; Hou, J. G.; Zhu, Q.; Chen, D. M. Low-Temperature Orientationally Ordered Structures of Two-Dimensional $\mathrm{C}_{60}$. J. Amer. Chem. Soc. 2003, 125, 169-172. 
66. Prato, M.; Lucchini, V.; Maggini, M.; Stimpfl, E.; Scorrano, G.; Eiermann, M.; Suzuki, T.; Wudl, F. Energetic preference in 5,6 and 6,6 ring junction adducts of $\mathrm{C}_{60}$ : fulleroids and methanofullerenes. J. Amer. Chem. Soc. 1993, 115, 8479-8480.

67. Porath, D.; Levi, Y.; Tarabiah, M.; Millo, O. Tunneling spectroscopy of isolated $\mathrm{C}_{60}$ molecules in the presence of charging effects. Phys. Rev. B 1997, 56, 9829-9833.

68. Neças, D.; Klapetek, P. Gwyddion: an open-source software for SPM data analysis. Centr. Eur. J. Phys. 2012, 10, 181-188.

69. Soler, J. M.; Artacho, E.; Gale, D., Julian; García, A.; Junquera, J.; Ordejón, P.; Sánchez-Portal, D. The SIESTA method for ab initio order-N materials simulation. J. Phys. Condens. Matter 2002, 14, 2745.

70. Kresse, G.; Hafner, J. Ab initio molecular dynamics for open-shell transition metals. Phys. Rev. B 1993, 48, 13115-13118.

71. Kresse, G.; Furthmüller, J. Efficient iterative schemes for ab initio total-energy calculations using a plane-wave basis set. Phys. Rev. B 1996, 54, 11169-11186.

72. Perdew, J. P.; Burke, K.; Ernzerhof, M. Generalized Gradient Approximation Made Simple. Phys. Rev. Lett. 1996, 77, 3865-3868.

73. Dion, M.; Rydberg, H.; Schröder, E.; Langreth, D. C.; Lundqvist, B. I. Van der Waals Density Functional for General Geometries. Phys. Rev. Lett. 2004, 92, 246401. 